Original Article

\title{
Investigation of long-term thermal aging-induced damage in oxide/oxide ceramic matrix composites
}

\author{
Zhengmao Yang ${ }^{\mathrm{a}, *}$, Junjie Yang ${ }^{\mathrm{b}}$ \\ ${ }^{a}$ Institute of Mechanics, Chinese Academy of Sciences, Beijing, China \\ ${ }^{\mathrm{b}}$ Institute of Aero engines, Tsinghua University, Beijing, China
}

\section{A R T I C L E I N F O}

\section{Keywords:}

Ceramic matrix composites

Thermal fatigue

Thermal aging-induced damage

Thermal aging

Hierarchical porosity

\begin{abstract}
A B S T R A C T
Long-term thermal aging is a typical factor affecting the thermo-mechanical fatigue life for hot-end components in the gas turbine. The present work focuses on the development of thermal aging-induced damage in 2-D woven oxide/oxide ceramic matrix composites from micro-mechanism and macroscopic mechanical performance. The porosity evolution and mechanical performance after long-term thermal aging were characterized through mercury intrusion measurements and uniaxial compressive tests, respectively. The results show that the decrease of micro-porosity directly reflects the irreversible evolution of material microstructure in the thermal aging process, and the decrease of compressive strength after aging is the macroscopic reflection of the microstructure variation. The porosity increment of matrix was thus used to characterize the thermal aging-induced damage, establishing a unique analysis model between the increment of micro-porosity under thermal aging and the corresponding degradation of material compressive strength. The experimental results are in good agreement with the established model.
\end{abstract}

\section{Introduction}

Ceramic matrix composites (CMCs) are considered to be attractive candidates for gas turbine engines, thermal protection systems of reentry vehicles and airframe structural components, due to their high melting point, good strength retention at high temperature, and excellent thermal shock resistance [1-3]. More essentially, oxide/oxide CMCs systems can withstand high-temperature oxidizing environments without any oxidation protective coating or cooling systems compared to $\mathrm{SiC} / \mathrm{SiC} \mathrm{CMCs}$. In addition, the superior fracture toughness properties of oxide/oxide CMCs ensure their strength and reliability in the presence of pores and notches, due to the built-in active stress redistribution mechanisms, thus minimizing the possibility of catastrophic brittle failure [4-6]. Therefore, oxide/oxide CMCs have been identified as the most promising class of materials for high-temperature industrial applications, where high structural efficiency is required under mechanical and thermal loadings. However, thermal aging may affect the mechanical behavior and fatigue performance of these composites. Consequently, investigation of the long-term thermal aging behavior of CMCs and quantitative description of the thermal aging-induced damage mechanism become of importance for the application of such composite structures.

The effects of the long-term thermal aging in air on CMCs has been extensively studied. Currently, commercially available oxide fibre reinforcement is limited to temperatures of $1000-1200^{\circ} \mathrm{C}$, depending on lifetime required. Furthermore, there has only been a small amount of research directed at investigating extended lifetimes at elevated temperatures. Xu et al. [7] determined the tensile strength of an $\alpha$-alumina fiber Nextel 610 after exposure to temperatures of 900, 1100 and $1300{ }^{\circ} \mathrm{C}$ using a two-parameter Weibull distribution. The result shows that the primary reasons for the loss of fiber strength are grain growth and defects induced during high-temperature exposure. Carelli et al. [8] investigated into the changes in the mechanical properties of an alloxide fiber-reinforced composite following long-term exposure $(1000 \mathrm{~h})$ at temperatures of $1000-1200{ }^{\circ} \mathrm{C}$ in air, and the result reveals that, despite the evidence of some strengthening of the matrix and the fibermatrix interfaces during aging, the vital tensile properties in the $0^{\circ} / 90^{\circ}$ orientation, including strength and failure strain, remains unchanged. Simon et al. [9] designed a porous mullite matrix to obtain the damagetolerant behavior as well as the high-temperature long-term stability. Kostopoulos et al. [10] investigated the anisotropic damage of alumina/ alumina continuous fiber ceramic composites under a corrosive/hightemperature exposure, in which stiffness matrix components and strength were experimentally defined as a function of exposure duration. Cluzel et al. [11] proposed a model of the mechanical behavior and lifetime of the CMCs with self-healing matrix, it is shown that the

\footnotetext{
* Corresponding author.

E-mail address: zmyang@imech.ac.cn (Z. Yang).
} 
coupling of mechanical and physicochemical mechanisms is a critical element in predicting the behavior of these materials in a structure subjected to complex thermo-mechanical solicitations in the oxidizing environment. Xu and Zok [12] presented analytical models for two potentially critical steps in a series of internal chemical and thermomechanical processes that lead to premature and localized fiber fracture for stress rupture of $\mathrm{SiC} / \mathrm{SiC}$ composites in intermediate-temperature oxidizing environments. Volkmann and Tushtev [13] analyzed the effects of heat treatments in the oxidative atmosphere on the mechanical and microstructural properties of a fiber-reinforced weak interface composite, and the results confirmed that the microstructural changes induced decreases the overall mechanical performance during hightemperature exposure. Subsequently, the micro-structures of Nextel ${ }^{\mathrm{TM}}$ 610 fibers in ceramic composites with different matrices, after processing and further heat treatment at $1300{ }^{\circ} \mathrm{C}$, were further studied to indicate the importance of microstructural investigations for CMCs [14]. Hay et al. [15] discussed the possible relationships among strength, grain-size and other causes of strength degradation after heat-treated at $1100-1500{ }^{\circ} \mathrm{C}$ from 1 to $100 \mathrm{~h}$ in air. Almeida et al. [16] analyzed the behavior of an oxide fiber at elevated temperatures before and after thermal shocks, which shows that the heat treatments increase the fiber stability and creep resistance, but reduce the tensile strength, and the changes observed in the creep strain versus duration time curves of the fibers are related to the observed microstructural transformations.

Unfortunately, more attention has been paid to the mechanical properties of the fiber after long-term aging, admittedly, the mechanical properties of the fiber phase are important, but the contribution of the matrix and fiber/matrix interface to the mechanical properties of the overall material has been neglected. In fact, in the early stage of material failure, the matrix and interface are the potential factors affecting the macroscopic mechanical properties of the composite structures. More essentially, plenty of literatures found that the work of the effect of the elevated temperature aging on the correlation among the microstructure of the material, mechanical behavior and thermo-mechanical mechanisms were barely carried out.

In the past two decades, extensive studies have been devoted to understand and describe the mechanical properties of CMCs $[17,18]$, but all the works did not form a quantitative description of the thermal aging-induced damage for CMCs fatigue life assessment at elevated temperature, as the knowledge or experience of the thermal aging treated CMCs is rather limited due to the complex microstructure and the complicated mechanical behavior of CMCs under thermal aging conditions.

The objective of the present work is to investigate the microstructural evolution and the mechanical behavior of the oxide/oxide CMCs after long-term thermal aging and to identify the thermal aginginduced damage mechanisms of the composites. The correlation between the microstructure and the macroscopic mechanical property is established based on detailed experimental observations and measurements. Then the thermal aging-induced damage evolution in the oxide/ oxide CMCs is characterized by the microstructural parameters and mechanical variables in the matrix.

\section{Materials and experiments}

\subsection{Material characterization}

In the present work, the tested material 2-D woven oxide/oxide CMCs consist of woven Nextel ${ }^{\mathrm{TM}} 610$ fibers $\left(99 \% \alpha-\mathrm{Al}_{2} \mathrm{O}_{3}\right)$, which are embedded in a $\mathrm{Al}_{2} \mathrm{O}_{3}-\mathrm{SiO}_{2}-\mathrm{ZrO}_{2}$ matrix with a density of $4.2 \mathrm{~g} /$ $\mathrm{cm}^{3}$. The uncoated Nextel ${ }^{\mathrm{TM}} 610$ fibers with a density of $4.0 \mathrm{~g} / \mathrm{cm}^{3}$ are in $0^{\circ} / 90^{\circ}$ woven layers. The fiber volume fraction of the CMCs is approximately $44 \%$, and the matrix contains chemical compositions of $85 \% \mathrm{Al}_{2} \mathrm{O}_{3}$ and $15 \% 3 \mathrm{YSZ}$ (in weight). The composites were produced using fabric type woven as 8 harness satin weave (HSW). The textile structures were impregnated with the paste-like $\mathrm{Al}_{2} \mathrm{O}_{3}$ slurry by knife
Table 1

Mechanical and thermal properties of the $\mathrm{Nextel}^{\mathrm{TM}} 610$ fiber and matrix in oxide/oxide CMCs at room temperature [1,19-21].

\begin{tabular}{llll}
\hline Materials phases & $\begin{array}{l}\text { TEC } \\
\left(10^{-6} /{ }^{\circ} \mathrm{C}\right)\end{array}$ & $\begin{array}{l}E \\
(\mathrm{GPa})\end{array}$ & $\begin{array}{l}\sigma_{\mathrm{u}} \\
(\mathrm{MPa})\end{array}$ \\
\hline $99 \% \alpha-\mathrm{Al}_{2} \mathrm{O}_{3}$ fiber & 8.8 & 373 & 2930 \\
$85 \% \mathrm{Al}_{2} \mathrm{O}_{3}-15 \%$ 3YSZ matrix & 10.2 & 210 & $\approx 45$ \\
\hline
\end{tabular}

blade coating. 8 layers were then stacked together, pressed and dried at $80^{\circ} \mathrm{C}-150^{\circ} \mathrm{C}$, the subsequent sintering process was conducted at $1100{ }^{\circ} \mathrm{C}-1300^{\circ} \mathrm{C}$, followed by infiltration with a 3YSZ-sol and a second sintering process at the same temperature for $5 \mathrm{~h}$.

The thermal expansion coefficient (TEC), elastic modulus, as well as the ultimate tensile strength of Nextel ${ }^{\mathrm{TM}} 610$ fiber and matrix are summarized in Table 1. Due to the high porosity of the material, exceeding $34 \%$, its effective property must be much lower than that in Table 1.

The as-received material is $2.8 \mathrm{~mm}$ thick plates. The plates of Nextel $^{\mathrm{TM}} 610$ having $0^{\circ} / 90^{\circ}$ fabric with lay-up, were machined into the specimens of coupon shape using the water jet. It should be noted that specimen dimensions are determined because of the limitations in manufacture processing, but the minimum dimension for each specimen is larger than the critical size that is required to ensure that the results are not dependent on specimen size.

Scanning electron microscope (SEM, JSM-7001F, Japan) micrograph of cross-section through the as-received composite can be seen in Fig. 1(a). Numerous irregular small matrix micro-cracks exist on the matrix-rich regions between fiber tows, those cracks are perpendicular to the layers of fibre reinforcement, which caused by the mismatch in TEC of fiber and matrix, and the constrained excessive shrinkage of the matrix during the material fabrication. Such micro-cracks are generally concentrated in the interface with the longitudinal fibers (oriented perpendicular to the crack plane) and penetrate only slightly into the transverse tows, as illustrated in Fig. 1(a). The voids arising during material processing can be seen in the center of Fig. 1(a). More essentially, the mesoscopic picture of the cutting surface reveals that there are a lot of cylindrical pores in size of $>100 \mu \mathrm{m}$ diameters oriented in the fiber direction and distributed in the thickness. It can be seen that the CMCs contain microscopic voids distributed overall, which can be interpreted as initial defects.

The fibers are generally assumed to be dense compared with matrices, as shown in Fig. 1(b), and there exist micropores among the fibre bundles.

The matrix is porous, as shown in Fig. 1(c). There exist micro-voids in the dimension of microns. The voids in the matrix, however, are much smaller than the matrix particles and less than $50 \mathrm{~nm}$ in size. The

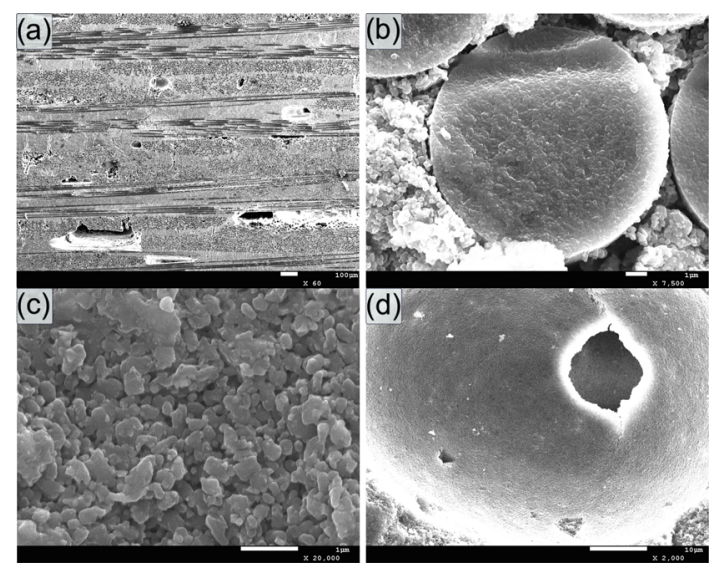

Fig. 1. Microstructure of the as-received 2-D woven oxide/oxide CMCs. (a) Overview, (b) fiber and matrix and (c) the porous matrix and (d) the spherical void. 
matrix is an effective collection of the alumina particles, if the particles are approximated as ellipsoids, the porosity of micro-pores around the ellipsoids in matrix can be calculated as $48 \%$. As the matrix volume fraction is $56 \%$, the micro-pores in matrix account for $28 \%$ of the material volume, which means that there are ca. $7 \%$ micro-pores in fiber plies [20].

Besides, the smooth uniform microstructure of pore surface in Fig. 1(d) reveals that the pore exists before cutting, the smooth sided nature of spherical void suggests that it has formed as a result of gas entrapment in the matrix slurry during matrix infiltration. The spherical nature of the large voids and their relatively smooth surface can be seen at higher magnification in Fig. 1(d), which support that these voids have been created by gas trapped with the matrix infiltrated fibre preform.

Combined with the above microstructure observation, the pores in the 2-D woven oxide/oxide CMCs can be divided into two different classes, so called "Hierarchical porosity" [20,22]: (i) the nanopores only exist in the matrix among the matrix particles, with a size less than $200 \mathrm{~nm}$, and (ii) the sintering shrinkage cracks and the micropores between the fiber filaments inside the fiber tow, with a size larger than $200 \mathrm{~nm}$, are presented as manufacturing micropores, which are typical for the oxide/oxide CMCs.

\subsection{Thermal aging tests}

Currently, commercially available oxide fibre reinforcement is limited to temperatures of $1000-1200{ }^{\circ} \mathrm{C}$, depending on lifetime required. Consequently, the specimens were isothermally exposed to a high-temperature environment at $1200{ }^{\circ} \mathrm{C}$ for $100-500 \mathrm{~h}$ to simulate the working conditions of gas turbines. As for the extreme environmental problems such as thermal overload that may occur during service, it is without the scope of the present work.

Samples to be thermal aged were placed in a crucible and subsequently positioned in the center of the furnace. After heating from room temperature to a temperature of $900{ }^{\circ} \mathrm{C}$ at a rate of $10^{\circ} \mathrm{C} / \mathrm{min}$, the samples were held for $30 \mathrm{~min}$ to stabilize the furnace temperature, and then further heated to the final aging temperature of $1200^{\circ} \mathrm{C}$ at the same rate. Once the samples had been aged for the appropriate time at $1200{ }^{\circ} \mathrm{C}$, the furnaces were cooled at a rate of $5{ }^{\circ} \mathrm{C} / \mathrm{min}$. Finally, the power to the elements was automatically switched off when the furnace reached a temperature of $200^{\circ} \mathrm{C}$, and the furnace was naturally cooled to room temperature, to avoid thermal shock effects for the oxide/oxide CMCs [23]. Note that all of the samples subjected to the thermal aging temperature at $1200{ }^{\circ} \mathrm{C}$ in the furnace should be simultaneously placed in the furnace zone at room temperature.

For every exposure duration, each set of specimens was subjected to a series of tests. All the samples were inspected to ascertain their initial state and quality as a reference state for each specimen, which was also a prerequisite for the subsequent evaluation of the test results.

On completion of the thermal aging treatments, the microstructure of samples was characterized principally using SEM to examine the microstructural evolution of composites after thermal aging and to investigate the thermal aging-induced damage mechanism.

\subsection{Mechanical testing}

In order to quantify the effects of different thermal aging duration on the mechanical properties of materials, in-plane mechanical properties of as-received composites and thermal aged composites were investigated through uniaxial monotonic compressive loadings in $0^{\circ}$ (weft) orientations.

All mechanical tests were performed at room temperature by using a servo-hydraulic axial/torsion material testing machine (MTS, Bionix, USA). Block specimens of $20 \mathrm{~mm} \times 30 \mathrm{~mm} \times 2.8 \mathrm{~mm}$ (thickness) with a gauge length of $15 \mathrm{~mm}$ were tested under compression [20]. The tests were performed with a constant displacement rate of $0.06 \mathrm{~mm} \cdot \mathrm{min}^{-1}$, to ensure quasi-static loading. The strain was measured with strain gage rosettes and calibrated with digital image correlation (DIC, VIC-3D SR$9 \mathrm{M}$, USA). Due to the restrictions on material supply, it is only possible to produce five samples from five different plates for each particular aging state.

\section{Microstructural evolution during thermal aging}

\subsection{Variations of the materials dimensions and density}

The density of oxide/oxide CMC specimens after thermal aging was calculated by using Archimedes immersion principle. The density of the specimen, through determining the specimens weighed in air and weighed in distilled water, is calculated as,

$\rho=\frac{W_{\mathrm{a}} \cdot \rho_{\mathrm{d}}}{W_{\mathrm{a}}-W_{\mathrm{d}}}$

where $\rho$ denotes the specimen density, $\rho_{d}$ is the density of distilled water, $W_{\mathrm{a}}$ and $W_{\mathrm{d}}$ are the weight of the specimen in air and distilled water, respectively.

The normalized thickness size and normalized density of oxide/ oxide CMCs treated at thermal aging at different periods are shown in Fig. 2. It indicates that there is a gradual decrease in the throughout the thickness size (perpendicular to the direction of the woven fibers) of the thermal aging treated oxide/oxide CMCs. This can be attributed to the fact that the material, perpendicular to the fiber-woven layer, is subjected to less constraint, so there is more freedom for the material to density in this direction. It is also worth noting that the 2-D woven fiber structure architecture restricts the material densification within the plane of the ply, and the physical size of the material in the two directions (fiber warp/weft direction) is not found to be reduced.

The change in oxide/oxide CMCs thickness (reduction) during it is exposed in thermal environments has important implications for the use of porous matrix CMCs, especially for the applications with high dimensional tolerance requirements (changes in component dimensions in turbine engines may result in changes in heat engine efficiency), the change in dimensions must be considered during the design steps.

\subsection{Variations of the hierarchical porosities during thermal aging}

Hierarchical porosities as well as generate pore size distribution of the open porosity both before and after aging were measured by using a porosimeter (Micromeritics, Autopore IV 9510, USA).

Fig. 3(a) illustrates the distribution of pores for four different CMC

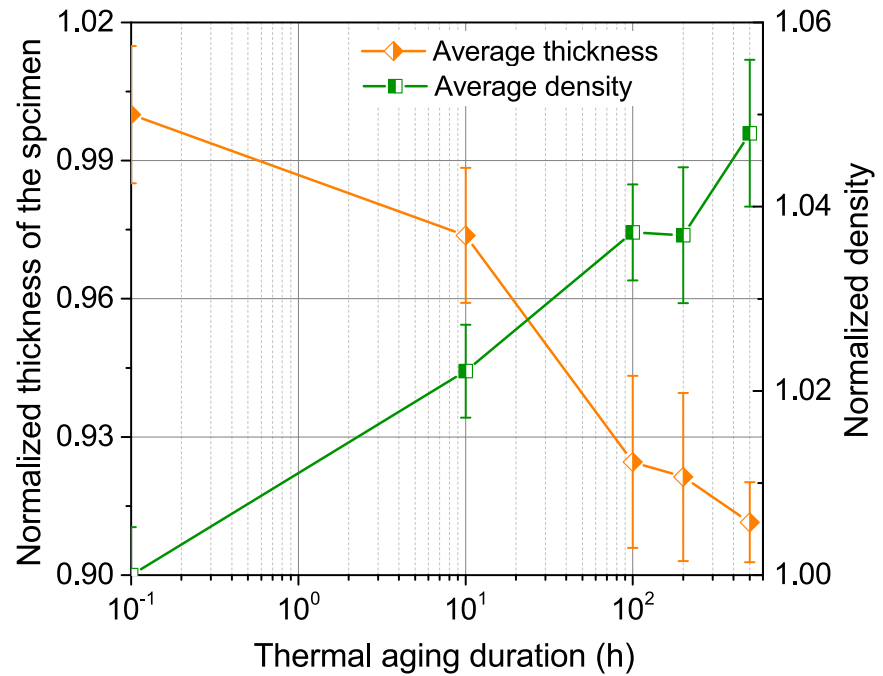

Fig. 2. The normalized density and thickness of specimen vs. the thermal aging duration $\left(T_{\text {aging }}=1200{ }^{\circ} \mathrm{C}\right)$. 


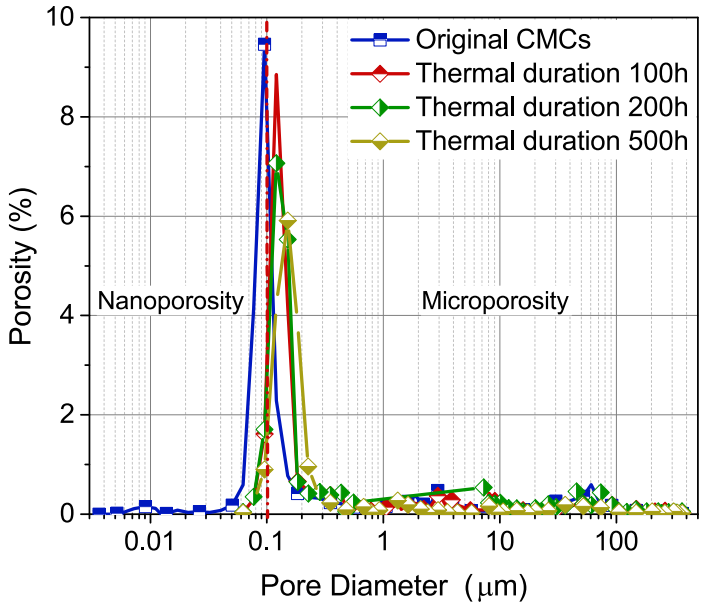

(a) Porosity vs. pore diameter.

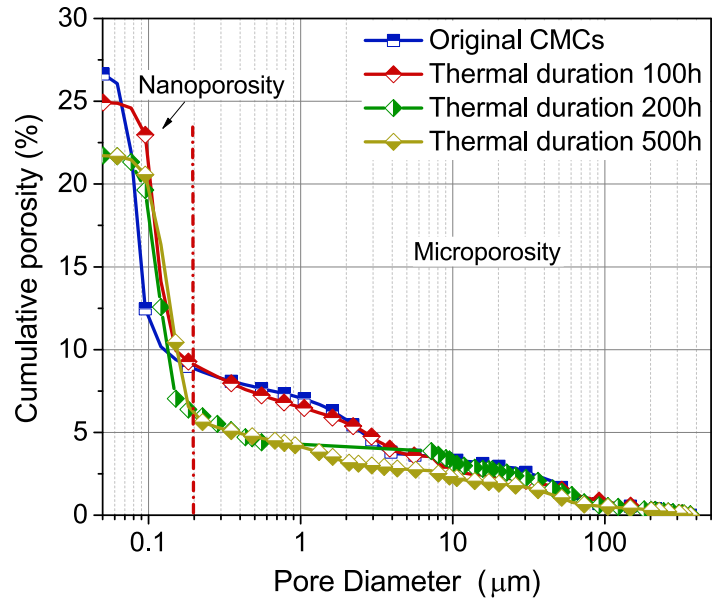

(b) Cumulative porosity vs. pore diameter.

Fig. 3. Pore size distributions for long-term thermal aged specimens determined by the mercury intrusion porosimetry.

states. All measurements reveal a large peak in the pore distribution at ca. $100 \mathrm{~nm}$, which corresponds to the pores between porous matrix particles. Interestingly, the peak for all thermal aged specimens is shifted right slightly, which means the small size decrement in the nano-voids. It can be concluded that as the aging time increases, the nano-pores decrease due to sintering between the matrix particles. In all specimens, the nano-porosity takes the major part in the total porosity, as shown in the accumulative porosity distribution in Fig. 3(b).

Figs. 3 and 4 elucidate that there are some irreversible microstructure changes in the matrix or interface during long-term thermal aging processing:

(i) The matrix particles may be sintered, as shown in Fig. 4(a), which reduces the porosity in the matrix-rich region. This interesting phenomenon is supported by the measurements of the composite density.

(ii) Bonding increases at the fibre/matrix interface and bridging reinforcement occurs, as illustrated in Fig. 4(b). In comparison, for the original composites, the bridging phenomenon was not observed by detailed SEM observation.

(iii) The partial of micro-cracks closure and pores filling are caused by the thermal aging treatment process, thus resulting in the composite densification.

The presence of hierarchical porosity is common in the oxide/oxide CMCs and has been reported in other studies [22,24]. However, there still are some thorny problems remained unsolved for the characterization of evolutions and the effect of hierarchical porosity on the mechanical properties of the composite structure.

Fig. 3(b) reveals that the porosity of materials decreases with accumulate thermal aging duration, which implies irreversible microstructural changes in the oxide/oxide composites. The fiber grain growth results in lower strength and stiffness values under long-term high temperature exposure. Although grain growth plays an essential

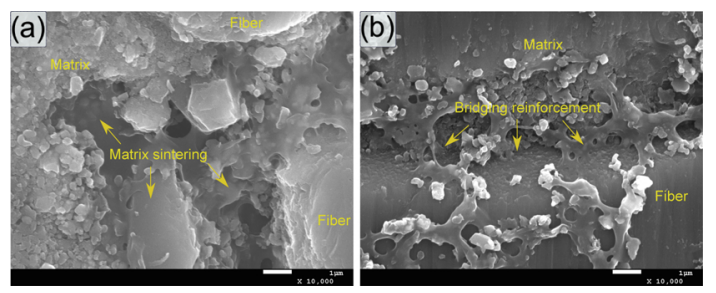

Fig. 4. Microstructure of the oxide/oxide CMCs after thermal aging duration for 500 h. (a) Matrix sintering and (b) fibers/matrix bridging reinforcement. role in fiber degradation, it is not the only microstructural change that occurs. Schmücker's detail experiments confirmed additionally that the microstructure and mechanical property of Nextel ${ }^{\mathrm{TM}} 610$ /alumina fibers are stable up to $1200{ }^{\circ} \mathrm{C}$, even in water vapour environment $[25,26]$. That is, the deterioration of the present oxide/oxide CMC is dominated by the matrix damage, whereas the fibers remain undamaged. Consequently, in order to quantify the microstructure evolution of the oxide/ oxide CMCs under thermal aging, as suggested in [20], the variation of the porosity can be expressed by

$\Delta \zeta=\zeta-\zeta_{0}$

where $\zeta$ is the current porosity and $\zeta_{0}$ is the initial porosity of composites; $\Delta \zeta$ is the porosity increment, representing material microstructural evolution. Since there are two different populations of pores which represent the evolution of hierarchical porosity, $\zeta$ denotes both nano- and micro-pores, and $\zeta$ is a monotonic function of the thermal aging duration.

Different porosities of the whole material plotted as functions of thermal aging duration are shown in Fig. 5. The decrement of the micro-pores can be approximated by a logarithmic function of thermal aging duration,

$\Delta \zeta=\alpha \zeta_{0}[\exp (-\mathrm{bt})-1]$

which implies increasing of material damage in the matrix. Above $\alpha$ and

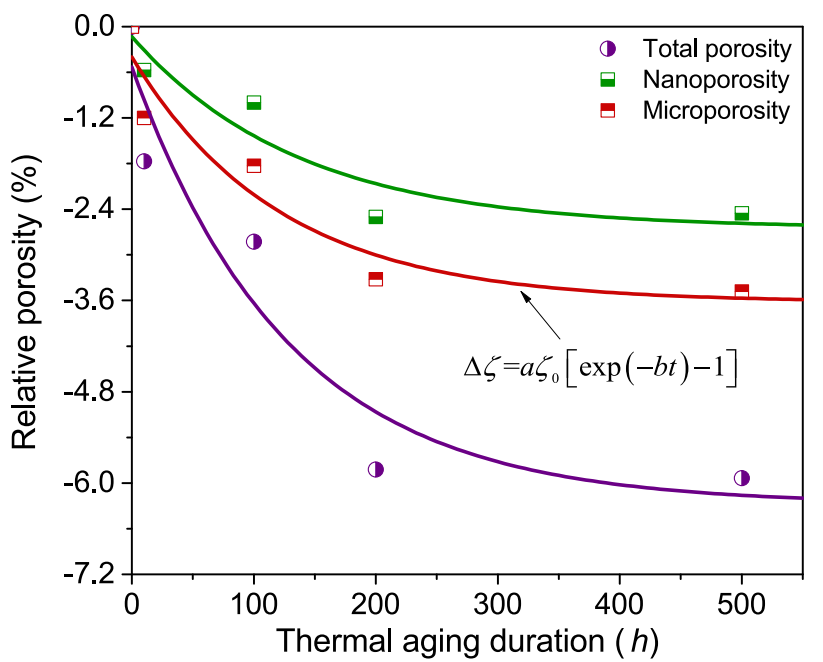

Fig. 5. Porosities of oxide/oxide CMCs as functions of the thermal aging duration $t$ (for the elevated temperatures equal to $1200{ }^{\circ} \mathrm{C}$ ). 


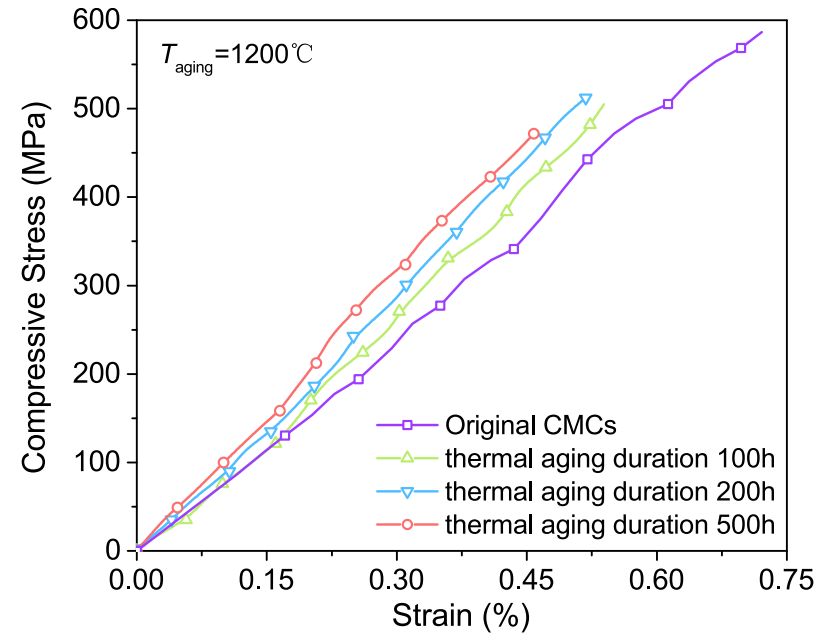

Fig. 6. Representative stress-strain curves for the as-received and the thermalaged specimens in the $0^{\circ}$ orientation.

$b$ are the fitting parameter of the approximation. Eq. (3) describes the porosity as a function of an increase in cumulative thermal aging duration. As shown in Fig. 5, the evolution of relative porosity with thermal aging duration can be divide into two stage: in the initial aging stage, the porosity decreases rapidly with increasing cumulative thermal aging duration, due to the partial of micro-cracks closure and pores filling; When the cumulative duration reaches ca. $200 \mathrm{~h}$, the porosity reaches a saturation phase. The total porosity increment is the sum of the nano-porosity and micro-porosity.

\section{Description of the thermal aging-induced damage}

\subsection{Evolution of material degradation}

The stress-strain curve of oxide/oxide CMCs before and after aging are shown in Fig. 6. The original oxide/oxide CMCs were subjected to accumulation thermal aging duration for $500 \mathrm{~h}$, the total porosity $\zeta$ was reduced from $27.6 \%$ to $21.7 \%, \Delta \zeta=5.9 \%$. The significant changes were that the elastic modulus slightly increased by $5.15 \%$, the compressive strength $\sigma_{\mathrm{u}}$ and the failure strain $\varepsilon_{\mathrm{f}}$ of thermal aged CMCs had a downward trend with $\Delta \sigma_{\mathrm{u}}=-17.73 \%$ and $\Delta \varepsilon_{\mathrm{f}}=-27.17 \%$, respectively.

As illustrated in Fig. 6, the current compressive strength of oxide/ oxide CMCs decreases with thermal aging duration $t$ in air. The reduction trend for every thermal aging duration $t$ refers to a specific set of specimens, and the compressive strength and elastic modulus values are normalized according to their respective initial values, which account for the variability of composites properties.

Fig. 7 illustrates the correlation between the normalized compression performance and thermal aging duration $t$ in the $0^{\circ}$ direction of oxide/oxide CMCs under high temperature exposure. There is a degradation in the compressive strength of composites and a corresponding increase in compressive elastic modulus of materials for the thermal aging temperature of $1200^{\circ} \mathrm{C}$. With the thermal aging duration progresses, the matrix continues to be compacted and the fiber/matrix interface strength continues to increase that the elastic modulus is continuously increased, which is reportedly due to the extensive densification of the matrix and an attendant loss in damage tolerance [8].

The main reasons for the thermal aging-induced strength decrease in oxide/oxide CMCs are as follows: (i) Micro-porosity in the matrices changes the micro-crack propagation path within the composite structure during the thermal aging process. More essentially, porosity coarsening severely reduces the ability of the matrix to crack, which results in the reduction of crack resistance and its ultimate compressive strength; (ii) The sintering of fiber/matrix interface properties becomes

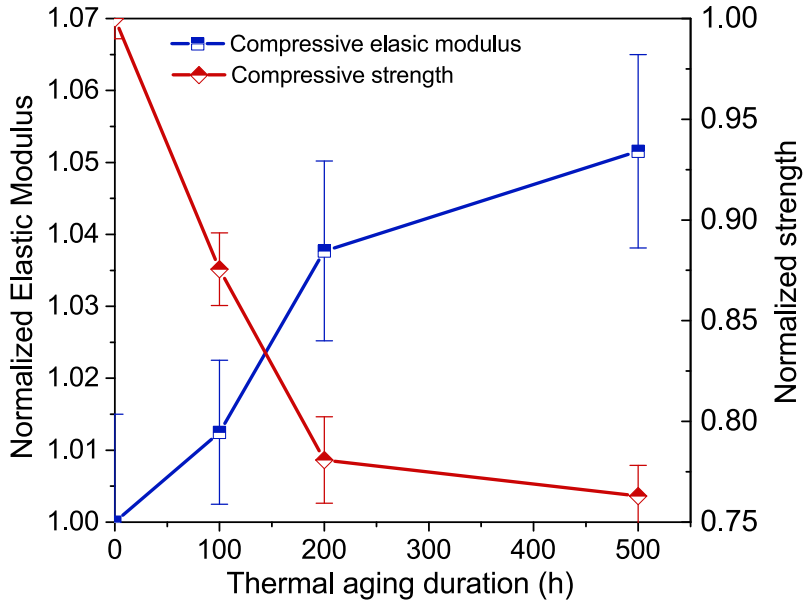

Fig. 7. Compressive mechanical properties as functions of the thermal aging duration $t$ for the elevated temperatures equal to $1200^{\circ} \mathrm{C}$.

possible, thereby reducing the peeling and pulling of the fibers, and the load redistribution ability of the fiber bundles is also deteriorated.

\subsection{Thermal aging-induced damage evolution}

In the framework of continuum damage mechanics, the nature of the damage is the reducing of the effective bearing area during the loading process, caused by micro-defects nucleating and expanding, and the damage can be represented by the decrease in the stiffness of the material. In the present work, the sintering of the matrix and the formation of bridges between fibers and matrix are observed during thermal aging process, which cannot be considered as the traditional "damage" mentioned above. However, these changes of microstructure lead to the strength of the composites decrease and an attendant loss in damage tolerance, which has been verified by experiments. Base on the above, the thermal aging-induced damage can be defined as the loss of the material strength [28],

$D_{\mathrm{TA}}=1-\frac{\sigma}{\sigma_{0}}$,

where $\sigma_{0}$ is the strength of undamaged material and $\sigma$ denotes the current strength of damaged material.

In the current work, a new damage model is extended from Kostopoulos's idea [10] and proposed based on the consideration of the effect of saturate damage to describe the thermal aging-induced damage evolution. The long-term thermal aging-induced damage $D_{\mathrm{TA}}$ is defined as,

$\frac{\mathrm{d} D_{\mathrm{TA}}}{\mathrm{d} t}=\xi f^{w}(\sigma, \varepsilon)\left(D_{\mathrm{f}}^{\mathrm{TA}}-D_{\mathrm{TA}}\right)^{-n}$

where $f^{w}(\sigma, \varepsilon)$ denotes the stress-strain state function of composites, $D_{\mathrm{f}}^{\mathrm{TA}}$ is the final saturation value of the thermal aging-induced damage for 2-D woven oxide/oxide CMCs obtained by the experiments, $\xi$ and $n$ are the material constants. Eq. (5) illustrates the aging thermal aginginduced damage as a non-linear function of the aging time. The stress-strain relationship of oxide/oxide CMCs under long-term high temperature exposure is linear elastic, so the damage rate $\left(\frac{\mathrm{d} D_{\mathrm{TA}}}{\mathrm{d} t}\right)$ at any time may be regarded as directly proportional to the thermal aginginduced damage $D_{\mathrm{TA}}$, and Eq. (5) can be simplified to

$\frac{\mathrm{d} D_{\mathrm{TA}}}{\mathrm{d} t}=-\xi\left(D_{\mathrm{f}}^{\mathrm{TA}}-D_{\mathrm{TA}}\right)$

Eq. (6) is a typical case of exponential function. However, the compression strength deterioration of composites did not continue until the failure of materials occurred as suggested in Eq. (6), but asymptotically approached a value, after which further high temperature exposure 
would no longer affect the mechanical performance of the composites. In other words, the high temperature exposure of 2-D woven oxide/ oxide CMCs to an isothermal $1200^{\circ} \mathrm{C}$ gas turbine combustion environment will never conclude to the final fracture of materials. The deterioration function of compressive strength may then be defined as:

$R(t)=\frac{\sigma(t)}{\sigma_{0}}$

where $\sigma(t)$ is the generalized compressive strength at any given time $t$ corresponding to the inverse of the diagonal elements of the compliance tensor and $\sigma_{0}$ and $\sigma_{\mathrm{f}}$ are the initial and final strength values. The compressive strength deterioration function $R(t)$ at any given time $t$ is in the scope of $[0,1]$ and $R(t)$ is defined as $R(t)=1-D_{\mathrm{TA}}$. In this case, Eq. (7) becomes:

$\frac{\mathrm{d} R}{\mathrm{~d} t}=-\xi\left[D_{\mathrm{f}}^{\mathrm{TA}}+R(t)-1\right]$

Integral of Eq. (8) for boundary conditions $R=1$ for $t=0$ yields

$R(t)=1-D_{\mathrm{f}}^{\mathrm{TA}}[1-\exp (-\xi t)]$

thus, the thermal aging-induced damage evolution of 2-D woven oxide/ oxide CMCs can be written as

$D_{\mathrm{TA}}=D_{\mathrm{f}}^{\mathrm{TA}}[1-\exp (-\xi t)]$

The correlation between long-term thermal aging-induced damage and thermal aging duration based on Eq. (10) are shown in Fig. 8, which indicates that long-term thermal aging-induced damage are determined by $D_{\mathrm{f}}^{\mathrm{TA}}$ and parameters $\xi$ that can be obtained by experiments.

\subsection{Correlation between porosity and material degradation}

The material degradation is a collective representation of irreversible microstructural changes. Porosity is an essential part of porous matrix CMCs due to the mixing of two or more different materials phases. It is well documented that the change of porosity under thermomechanical loading is one of the major factors influencing the mechanical performance of composite structures. Consequently, the establishment of a quantitative correlation between the porosity and damage in the corresponding state can contribute to an in-depth understanding for the mechanical behavior and application of porous matrix CMCs.

The mechanical properties are directly related to the porosity in powder metallurgy [27]. The correlation between the porosity and the mechanical property can be expressed as

$\frac{P}{P_{0}}=\left(\frac{\rho}{\rho_{0}}\right)^{k}$

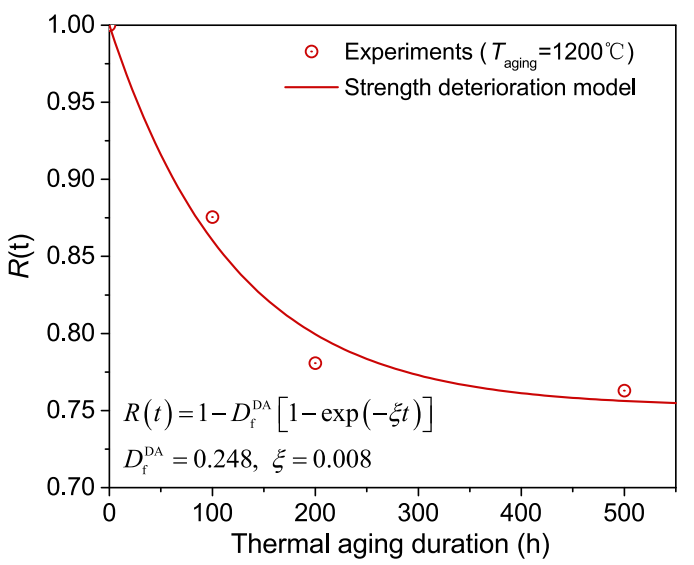

where $P$ as mechanical property (such as $E$ modulus, yield stress and fatigue limit) with the mass density $\rho ; P_{0}$ denotes the mechanical property of the reference material with the density $\rho_{0}$. The exponent $k$ is an empirical fitting parameter.

In porous matrix CMCs, the mechanical properties of composites are expected to decrease with increasing porosity. Consequently, the power-law relationship has been extended to damage modeling [20], the compressive strength of 2-D woven oxide/oxide CMCs is selected as a parameter to characterize the degradation of the composites mechanical properties induced by long-term thermal aging. Then, the current compressive strength of materials with a porosity of $\zeta$ can be rewritten as the corresponding state.

$\frac{\sigma}{\sigma_{0}}=\left(\frac{1-\zeta}{1-\zeta_{0}}\right)^{q}=\left(1-\frac{\zeta-\zeta_{0}}{1-\zeta_{0}}\right)^{q}=\left(1-\frac{\Delta \zeta}{1-\zeta_{0}}\right)^{q}$

with $q$ as the fitting parameter. Above the reference state is the initial CMC with porosity $\zeta_{0}$.

From Eqs. (4) and (12), the long-term thermal aging-induced damage evolution of CMCs can be expressed by the porosity increment of the micro-porosity in the corresponding state of materials, $\Delta \zeta$,

$D_{\mathrm{TA}}=1-\left(1-\frac{\Delta \zeta}{1-\zeta_{0}}\right)^{q}$.

Eq. (13) indicates that the long-term thermal aging-induced damage of CMCs can be represented by the porosity increment of the material micro-porosity in the corresponding state, which also follows the power function relationship.

From Eqs. (3) and (10), the long-term thermal aging-induced damage evolution can be obtained by the porosity increment $\Delta \zeta$ of composites in the corresponding state,

$D_{\mathrm{TA}}=\frac{D_{\mathrm{f}}^{\mathrm{TA}}}{\alpha \zeta_{0}} \Delta \zeta$

Eq. (14) elucidates a simple linear relationship between thermal aginginduced damage and its corresponding porosity. More essentially, the long-term thermal aging-induced damage of 2-D woven oxide/oxide CMCs can be predicted by the porosity in its corresponding state.

The linear relationship between the compressive strength of thermal aged oxide/oxide CMCs in the $0^{\circ}$ direction and the relative porosity $\Delta \zeta$ is shown in Fig. 9(a). The power function model and the proposed linear model are well predicted with experimental data. The range of porosity increment studied in this present work is $\Delta \zeta<4 \%$, as the maximum porosity increment of oxide/oxide CMCs micro-pores is at most $4 \%$ at the current experimental high temperature exposure duration of $500 \mathrm{~h}$.

Fig. 9(b) gives a relationship between the long-term thermal aginginduced damage and its corresponding micro-porosity increment $\Delta \zeta$. It can be observed that both thermal aging-induced damage evolution

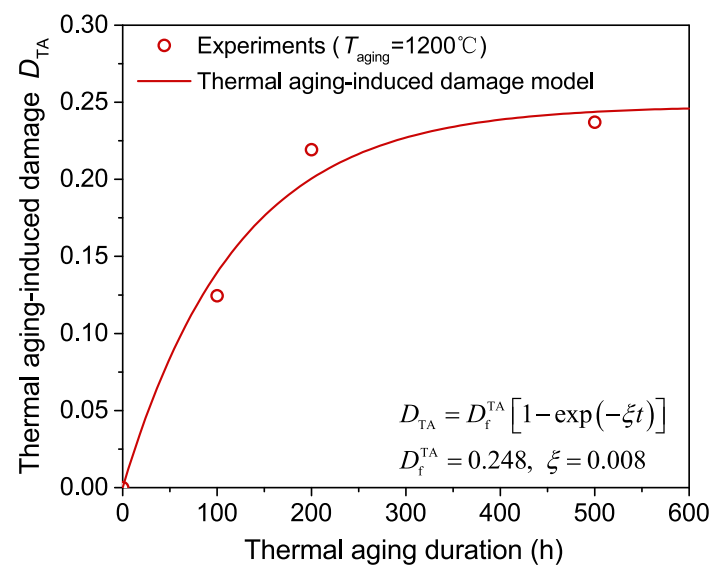

Fig. 8. Correlation between long-term thermal aging-induced damage and thermal aging duration $t$ in oxide/oxide CMCs (aging temperature $T_{\text {aging }}=1200{ }^{\circ} \mathrm{C}$ ). 

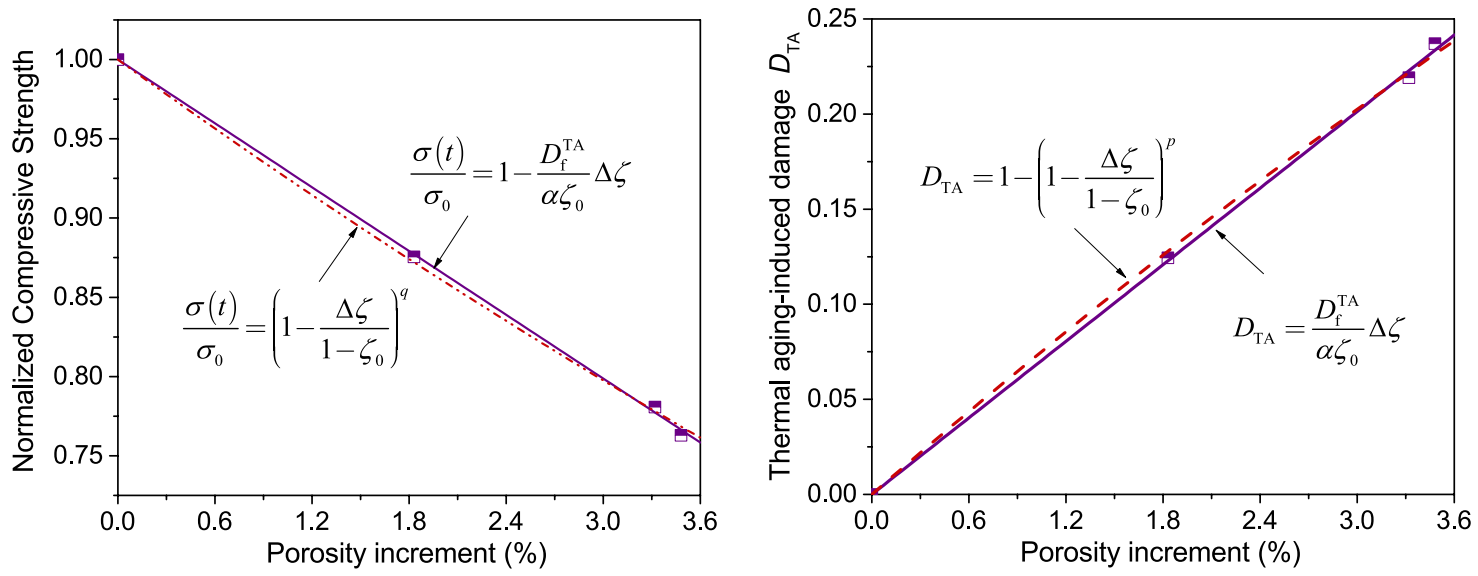

Fig. 9. Correlations between the mechanical property and the porosity.

models (power function model and linear model proposed in present work) have a good correlation with experimental data, which indicates that both of them can well predict the thermal aging-induced damage of oxide/oxide CMCs. However, the linear model (Eq. (14)) provides a much more clear physical meaning that the thermal aging-induced damage is linearly correlated with the micro-porosity increment. More essentially, the relatively good agreement between experiment and the power law/linear law, based on the simple ellipsoids pore geometry assumptions, elucidates that the effect of the shape and morphology of micro-porosity on the mechanical performance of 2-D woven oxide/ oxide CMCs is not apparent.

\section{Conclusions}

In the present work, the thermal aging-induced damage evolution of 2-D woven oxide-oxide CMCs has been investigated experimentally. Based on detailed experimental observations, an evolution equation for the thermal aging-induced damage has been established and verified. From the experimental results, the following conclusions can be drawn:

- Evolution of the micro-porosity in the matrix can provide a more precise description of the overall thermal aging-induced damage process.

- A novel model was presented to describe the thermal aging-induced damage evolution for oxide/oxide CMCs. It is confirmed that the thermal aging-induced damage is a function of during times, and the phenomenological thermal aging-induced damage can be characterized by the degradation of the material compressive strength, which can be quantitatively described by the damage model.

- Based on experimental observations, thermal aging-induced damage evolution is established and verified. The phenomenological damage model predicts that the thermal aging-induced damage is linearly correlated with the micro-porosity increment, and the model parameters can be identified from thermal aging tests.

\section{Conflicts of interest}

The authors declare no conflicts of interest.

\section{Acknowledgement}

The present work is supported by the Strategic Priority Research Program of Chinese Academy of Sciences (Grant No. XDA17030100), the National Science Foundation of China (Grant No. 11572169 and 51775294).

\section{References}

[1] D.M. Wilson, L.R. Visser, High performance oxide fibers for metal and ceramic composites, Compos. Part A: Appl. Sci. Manuf. 32 (8) (2001) 1143-1153.

[2] M.B. Ruggles-Wrenn, A.T. Radzicki, S.S. Baek, K.A. Keller, Effect of loading rate on the monotonic tensile behavior and tensile strength of an oxide-oxide ceramic composite at $1200{ }^{\circ} \mathrm{C}$, Mater. Sci. Eng. A 492 (1-2) (2008) 88-94.

[3] J. Lamon, Review: creep of fibre-reinforced ceramic matrix composites, Int. Mater. Rev. (2019) 1-35.

[4] H. Richter, P.W.M. Peters, Tensile strength distribution of all-oxide ceramic matrix mini-composites with porous alumina matrix phase, J. Eur. Ceram. Soc. 36 (13) (2016) 3185-3191.

[5] F. Langlais, G.L. Vignoles, 5.4 Chemical Vapor Infiltration Processing of Ceramic Matrix Composites, Elsevier, Oxford, 2018, pp. 86-129.

[6] Z. Yang, H. Liu, Effects of thermal aging on the cyclic thermal shock behavior of oxide/oxide ceramic matrix composites, Mater. Sci. Eng.: A 769 (2020) 138494.

[7] Z.R. Xu, K.K. Chawla, X. Li, Effect of high-temperature exposure on the tensilestrength of alumina fiber Nextel 610, Mater. Sci. Eng. A 171 (1-2) (1993) 249-256.

[8] E.A.V. Carelli, H. Fujita, J.Y. Yang, F.W. Zok, Effects of thermal aging on the mechanical properties of a porous-matrix ceramic composite, J. Am. Ceram. Soc. 85 (3) (2002) 595-602.

[9] R.A. Simon, Progress in processing and performance of porous-matrix oxide/oxide composites, Int. J. Appl. Ceram. Technol. 2 (2) (2005) 141-149.

[10] V. Kostopoulos, D.E. Vlachos, A. Paipetis, G. Sotiriadis, Anisotropic damage of alumina/alumina CFCCs under long term high temperature exposure: investigations by ultrasonic stiffness measurements and quasi-static tests, Compos. Sci. Technol. 66 (16) (2006) 3221-3229.

[11] C. Cluzel, E. Baranger, P. Ladeveze, A. Mouret, Mechanical behaviour and lifetime modelling of self-healing ceramic-matrix composites subjected to thermomechanical loading in air, Compos Part A - Appl. Sci. Manuf. 40 (8) (2009) 976-984.

[12] W. Xu, F.W. Zok, R.M. McMeeking, Model of oxidation-induced fiber fracture in SiC/SiC composites, J. Am. Ceram. Soc. 97 (11) (2014) 3676-3683.

[13] E. Volkmann, K. Tushtev, D. Koch, C. Wilhelmi, G. Grathwohl, K. Rezwan, Influence of fiber orientation and matrix processing on the tensile and creep performance of Nextel 610 reinforced polymer derived ceramic matrix composites, Mater. Sci. Eng. A 614 (Suppl. C) (2014) 171-179.

[14] E. Vokmann, M.D. Barros, K. Tushtev, W.E.C. Pritzkow, D. Koch, J. Goring, C. Wilhelmi, G. Grathwohl, K. Rezwan, Influence of the matrix composition and the processing conditions on the grain size evolution of Nextel 610 fibers in ceramic matrix composites after heat treatment, Adv. Eng. Mater. 17 (5) (2015) 610-614.

[15] R.S. Hay, K.A. Keller, L.P. Zawada, N.S. Jacobson, G.E. Fair, Degradation of Nextel ${ }^{\mathrm{TM}}$ 610-based oxide-oxide ceramic composites by aluminum oxychloride decomposition products, J. Am. Ceram. Soc. 101 (9) (2018) 4203-4223.

[16] R.S.M. Almeida, E.L. Bergmuller, H. Luhrs, M. Wendschuh, B. Clauss, K. Tushtev, K. Rezwan, Thermal exposure effects on the long-term behavior of a mullite fiber at high temperature, J. Am. Ceram. Soc. 100 (9) (2017) 4101-4109.

[17] P.E. Cantonwine, Strength of thermally exposed alumina fibers. Part II: Bundle behavior, J. Mater. Sci. 38 (3) (2003) 471-480.

[18] A.M. Parvanian, H.R. Salimijazi, M. Fathi, M. Saadatfar, Synthesis and thermal shock evaluation of porous SiC ceramic foams for solar thermal applications, J. Am. Ceram. Soc. 0 (0) (2018).

[19] B. Moser, A. Rossoll, L. Weber, O. Beffort, A. Mortensen, Nextel ${ }^{\mathrm{TM}} 610$ alumina fibre reinforced aluminium: influence of matrix and process on flow stress, Compos. Part A: Appl. Sci. Manuf. 32 (8) (2001) 1067-1075.

[20] Z. Yang, H. Yuan, B. Markert, Representation of micro-structural evolution and thermo-mechanical damage in thermal shocked oxide/oxide ceramic matrix composites, Int. J. Fatigue 126 (2019) 122-129.

[21] Y. Yin, J. Zhang, Alumina Ceramics and Alumina Ceramics Matrix Composites, Chemical Industry Press, Beijing, 2001.

[22] Z. Yang, H. Liu, H. Yuan, Micro-porosity as damage indicator for characterizing 
cyclic thermal shock-induced anisotropic damage in oxide/oxide ceramic matrix composites, Eng. Fract. Mech. 220 (2019) 106669.

[23] Z. Yang, H. Yuan, H. Liu, Evolution and characterization of cyclic thermal shockinduced thermomechanical damage in oxide/oxide ceramics matrix composites, Int J. Fatigue 120 (2019) 150-161.

[24] C. Ben Ramdane, A. Julian-Jankowiak, R. Valle, Y. Renollet, M. Parlier, E. Martin, P. Diss, Microstructure and mechanical behaviour of a Nextel ${ }^{\mathrm{TM}}$ 610/alumina weak matrix composite subjected to tensile and compressive loadings, J. Eur. Ceram. Soc. 37 (8) (2017) 2919-2932.
[25] M. Schmücker, P. Mechnich, Improving the microstructural stability of Nextel ${ }^{\mathrm{TM}} 610$ alumina fibers embedded in a porous alumina matrix, J. Am. Ceram. Soc. 93 (7) (2010) 1888-1890.

[26] M. Schmücker, F. Flucht, P. Mechnich, Degradation of oxide fibers by thermal overload and environmental effects, Mater. Sci. Eng.: A 557 (2012) 10-16.

[27] P. Beiss, Pulvermetallurgische Fertigungstechnik, Springer, Berlin, Heidelberg, 2010.

[28] J. Lemaitre, R. Desmorat, Engineering Damage Mechanics: Ductile, Creep, Fatigue and Brittle Failures, Springer-Verlag Berlin Heidelberg, Berlin, Heidelberg, 2005. 